\title{
35
}

\section{An approach to infinite domains, singularities and superelements in FEM computations}

\author{
Antoni Żochowski \\ Systems Research Institute of the Polish Academy of Sciences \\ Newelska 6, 01-447 Warszawa, Poland
}

\begin{abstract}
In the paper the representation method for treating the infinite parts of computational domains is derived. It is based on a discrete formulation of the problems and uses the formal series technique. The method applies to wedge-like domains and static problems in general. For finite domains, it makes possible a uniform treatment of problems with corner singularities and improvement of accuracy in ordinary FEM computations.
\end{abstract}

\section{Keywords}

Finite elements, elliptic equations, infinite domains, singular solutions

\section{INTRODUCTION}

The method presented in this paper is quite general and may be used in finite element computations for partial differential equations or systems of any order (2-nd, 4-th or higher if it makes sense), but we shall concentrate here on the second order examples and plane problems.

Let us imagine a bounded star-shaped domain $\Omega_{s}$, having the center at $0 \in \mathrm{R}^{2}$, and define an exterior domain $\Omega_{e}=\mathrm{R}^{2}-\Omega_{s}$ with the boundary $\Gamma^{0}=\partial \Omega_{e}$. Next we approximate $\Gamma^{0}$ by $\Gamma_{h}^{0}$ using linear segments and extend a set of radii originating from 0 and going through nodes of $\Gamma_{h}^{0}$. Now we may define consecutive crossections of $\Omega_{e}$ by similarity transformation with center in 0 , taking $\Gamma_{h}^{i}=r^{i} \cdot \Gamma_{h}^{0}$, where $r>1$ and $i=0,1,2, \ldots$. Between $\Gamma_{h}^{i}$ and $\Gamma_{h}^{i+1}$ lay the ring-like parts of $\Omega_{e h}$, denoted by $\Omega_{e h}^{i}$. These rings are also similar, since $\Omega_{e h}^{i+1}=r \cdot \Omega_{e h}^{i}$, and they sum up to the whole $\Omega_{e h}$, giving in this way the discretization of the exterior of the "hole' in $\mathrm{R}^{2}$. It is obvious, that we may as well consider a part of $\Omega_{e h}$ limited by two radii, on which homogeneous Neumann or Dirichlet boundary conditions are imposed, while the loading of $\Gamma_{h}^{0}$ is arbitrary.

Our goal is to solve elasticity or Laplace equations in such a domain using finite element method. Let the parts between crossections $k, k+1$ be discretized with some kind of elements. We shall denote by $u_{k}$ the vector of all nodal values of solution corresponding to the $\mathrm{k}$-th crosssection, discounting those, which may be eliminated immediately due to 
the homogeneous Dirichlet conditions on the bounding radii. Now the elastic energy of the whole body after discretization can be written as

$E=\sum_{k=0}^{\infty} E_{k}\left(u_{k}, u_{k+1}\right)$,

where $E_{k}$ denotes the energy of the k-th ring. Let us concentrate on $E_{0}$. By eliminating internal nodes between sections $\Gamma^{0}$ and $\Gamma^{1}$ we get

$E_{0}\left(u_{0}, u_{1}\right)=\frac{1}{2}\left[u_{0}^{T}, u_{1}^{T}\right] \cdot M \cdot\left[\begin{array}{l}u_{0} \\ u_{1}\end{array}\right]$,

where $2 n \times 2 n$ symmetric stiffness matrix $M\left(n=\operatorname{dim} u_{k}\right)$ has the form

$M=\left[\begin{array}{lll}A_{1} & , & B \\ B^{T} & , & A_{2}\end{array}\right]$.

Observe, that both $n \times n$ matrices $A_{1}, A_{2}$ are symmetric and positive definite.

Now comes the crucial observation. Assume, that $\Omega_{e h}^{i}$ has been triangulated and the linear finite elements have been used. Then the matrix $M$ is proportional to the area of the ring, that is $r^{2}$, and inversely proportional to the squares of lengths of the sides of triangles, since the gradient of $u$ is inversely proportional to $r$. As a result, $M$ is the same for all rings, and $E_{k}$ have the same form for all $k$.

Using the above notation we may write the energy of the whole $\Omega_{e h}$ as

$E=\frac{1}{2}\left[\begin{array}{l}u_{0} \\ u_{1} \\ u_{2} \\ \vdots \\ \vdots\end{array}\right]^{T} \cdot\left[\begin{array}{lllll}A_{1} & B & & & \\ B^{T} & A & B & & \\ & B^{T} & A & B & \\ & & B^{T} & \ddots & \\ & & & & \ddots\end{array}\right] \cdot\left[\begin{array}{l}u_{0} \\ u_{1} \\ u_{2} \\ \vdots \\ \vdots\end{array}\right]$

where $A=A_{1}+A_{2}$. If we could express $E$ as a function of $u_{0}$ only, $E=E\left(u_{0}\right)$, then it would be possible to write down the whole energy in terms of finite number of nodal values.

The problem of representing the infinite body has received some attention in FEM literature. In (Givoli,1992),(Grote,Keller,1994) the use is made of the exact analytic solutions in certain kinds of infinite domains in order to get substitute boundary conditions. Here we treat the problem from the beginning in its discrete formulation and use energetic approach. In (Sharau,1994) a completely different method is used, depending on treating the infinite body as a certain kind of elastic support. The method presented here may be proved to be correct (convergent), but of course has also limitations. It applies to periodic or "radially" periodic 2-D and 3-D bodies with finite arbitrary part. Nevertheless, it may be generalized as well, as it will be shown later. 


\section{PROBLEM FORMULATION}

Let us return to the expression for $E$, (4), and solve the elasticity equations in the whole right part imposing the boundary conditions on $u_{0}$. The necessary condition for the minimum of energy takes on the form

$M_{\infty} \cdot u_{\infty}=\left[\begin{array}{lllll}A & B & & & \\ B^{T} & A & B & & \\ & B^{T} & A & B & \\ & & B^{T} & \ddots & \\ & & & & \ddots\end{array}\right] \cdot\left[\begin{array}{l}u_{1} \\ u_{2} \\ u_{3} \\ \vdots \\ \vdots\end{array}\right]=\left[\begin{array}{c}-B^{T} \\ 0 \\ 0 \\ \vdots \\ \vdots\end{array}\right] \cdot u_{0}$.

As a result, we must solve the matrix equation of the infinite order

$M_{\infty} \cdot Q_{\infty}=\left[\begin{array}{lllll}A & B & & & \\ B^{T} & A & B & & \\ & B^{T} & A & B & \\ & & B^{T} & \ddots & \\ & & & & \ddots\end{array}\right] \cdot\left[\begin{array}{l}Q_{1} \\ Q_{2} \\ Q_{3} \\ \vdots \\ \vdots\end{array}\right]=\left[\begin{array}{c}-B^{T} \\ 0 \\ 0 \\ \vdots \\ \vdots\end{array}\right]$

where $Q_{1}, Q_{2}, \ldots$ have dimensions $n \times n$.

It is well known (Cooke,1950), that such systems may have infinitely many solutions. Therefore we impose the physical condition, that the consecutive energy terms (2) diminish, or the requirement, that the necessary condition gives minimum, not the saddle point or maximum of the elastic energy. As it will turn out, this makes the solution unique.

If we could obtain the expression for $Q_{i}$ in the multiplicative form, $Q_{i}=Q^{i}$, then taking into account, that $u_{k}=Q_{k-1} u_{0}=Q^{k-1} u_{0}$, the energy on the whole domain takes on the form

$$
\begin{aligned}
E\left(u_{k}, u_{k+1}\right) & =\frac{1}{2}\left(u_{k}^{T} A_{1} u_{k}+u_{k+1}^{T} B^{T} u_{k}+u_{k}^{T} B u_{k+1}+u_{k+1}^{T} A_{2} u_{k+1}\right) \\
& =\frac{1}{2} u_{1}^{T} \cdot\left(Q^{T}\right)^{k} \cdot\left[A_{1}+Q^{T} B^{T}+B Q+Q^{T} A_{2} Q\right] \cdot Q^{k} \cdot u_{1} .
\end{aligned}
$$

Hence, assuming the convergence of the infinite sum, the whole $E$ may be computed as $E=\frac{1}{2} u_{0}^{T} \cdot S \cdot u_{0}$ where $S=\sum_{k=0}^{\infty}\left(Q^{T}\right)^{k} \cdot R \cdot Q^{k}, R=A_{1}+Q^{T} B^{T}+B Q+Q^{T} A_{2} Q$. The series for $S$ can be, as we shall see later, computed exactly in a closed form.

\section{FORMAL SERIES APPROACH}

In this section, we shall solve the equation (6) by embedding the problem into the framework of operations on infinite series (Stanley,1986). Let us establish the correspondence between the infinite vector $f_{\infty}=\left[f_{1}, f_{2}, \ldots\right]^{T}$ and the formal power series:

$f(x)=\sum_{i=1}^{\infty} f_{i} \frac{x^{i-1}}{(i-1) !}$. 
Differentiating this series gives

$$
D f(x)=\sum_{i=2}^{\infty} f_{i} \frac{x^{i-2}}{(i-2) !},
$$

or, in vector representation,

$$
f_{\infty}=\left[f_{1}, f_{2}, \ldots\right]^{T}, \quad D f_{\infty}=\left[f_{2}, f_{3}, \ldots\right]^{T} .
$$

This shows, that the differentiation may be represented as multiplication by the matrix:

$$
D f_{\infty}=\left[\begin{array}{ccccc}
0 & 1 & & & \\
& 0 & 1 & & \\
& & 0 & 1 & \\
& & & \ddots & \ddots
\end{array}\right] \cdot f_{\infty} .
$$

and similarly for integration. Let us notice, that $M_{\infty}$ has a block structure, i.e. every n-th row repeats itself after shifting $n$ places to the right. Therefore we must introduce a whole vector of functions

$$
w^{k}(x)=\sum_{j=1}^{\infty} w_{j}^{k} \frac{x^{j-1}}{(j-1) !}, \quad k=1, \ldots, n,
$$

and denote $u_{i}=\left[w_{i}^{1}, \ldots, w_{i}^{n}\right]^{T}$, so that

$$
u(x)=\left[\begin{array}{c}
w^{1}(x) \\
\vdots \\
w^{n}(x)
\end{array}\right]=\sum_{i=1}^{\infty} u_{i} \frac{x^{i-1}}{(i-1) !} .
$$

If we neglect first $n$ rows, the system (6) is equivalent to $B^{T} \cdot \int u+A \cdot u+B \cdot D u=0$, and putting $\bar{u}=\int u$ gives finally the differential equation $B \cdot \bar{u}^{\prime \prime}+A \cdot \bar{u}^{\prime}+B^{T} \cdot \bar{u}=0$. The solution must have the form $\bar{u}=r_{\lambda} \cdot e^{\lambda x}$, where $\operatorname{dim} r_{\lambda}=n$. Furthermore, $\lambda$ is the root of the $2 \mathrm{n}$-th order characteristic polynomial

$\operatorname{det}\left(B \cdot \lambda^{2}+A \cdot \lambda+B^{T}\right)=0$

and $r_{\lambda}$ should be the right eigenvector: $\left(B \cdot \lambda^{2}+A \cdot \lambda+B^{T}\right) \cdot r_{\lambda}=0$. In general, (13) has $2 \mathrm{n}$ roots. However, from the particular form of (13) it follows, that these roots occur in pairs, $\left(\lambda_{i}, 1 / \lambda_{i}\right), i=1, \ldots n$. Let us eliminate at this point the roots with absolute values bigger than 1 , and consider (after rearranging) only $\lambda_{1}, \ldots \lambda_{n}$. The corresponding solutions have the form:

$\bar{u}=c_{1,1} r_{\lambda_{1}} \exp \left(\lambda_{1} x\right)+c_{2,1} r_{\lambda_{2}} \exp \left(\lambda_{2} x\right)+\ldots+c_{n, 1} r_{\lambda_{n}} \exp \left(\lambda_{n} x\right)$.

The constants $c_{1,1}, \ldots, c_{n, 1}$ are chosen in such a way, that the first $n$ rows of $(6)$ are satisfied. Let us notice, however, that equation (6) has $n$ right-hand sides. Thats why we have double subscript here: $c_{p, q}$ denotes $\mathrm{p}$-th constant corresponding to the $\mathrm{q}$-th column on the right. 
Let us now introduce the following notation: $R_{\lambda}=\left[r_{\lambda_{1}}, \ldots, r_{\lambda_{n}}\right], C=\left[c_{j, k}\right]_{j, k=1, \ldots n}, \Lambda=$ $\operatorname{diag}\left[\lambda_{1}, \ldots, \lambda_{n}\right]$. It may be proved, that the solution of (6) takes on the form $Q_{1}=$ $R_{\lambda} \cdot \Lambda \cdot C, Q_{2}=R_{\lambda} \cdot \Lambda^{2} \cdot C, \ldots, Q_{n}=R_{\lambda} \cdot \Lambda^{n} \cdot C$. The choice of the matrix of constants $C$ must ensure satisfaction of the first block of equations, what leads to the formula $C=R_{\lambda}^{-1}$. In consequence we get, as was required: $Q_{1}=Q=R_{\lambda} \cdot \Lambda \cdot R_{\lambda}^{-1}, \quad Q_{i}=$ $Q^{i}=R_{\lambda} \cdot \Lambda^{i} \cdot R_{\lambda}^{-1}, \quad i=1,2, \ldots$ In general there may appear $\lambda=1$, what corresponds to the constant solution. For scalar Laplace equation it must have multiplicity 2 , with only one eigenvector. For elasticity system, there exist pairs consisting of eigenvalue $\lambda=1$ and eigenvector responsible for constant displacements in 2 or 3 independent directions (rotations are excluded, since they imply $\lambda>1$ ). However, they do not contribute to the energy, so the convergence of the energy series is assured by the next eigenvalue strictly smaller than 1 .

We may also prove, that after regrouping the terms, the matrix $S$ takes on the simple form: $S=A_{1}+B Q$.

\section{GENERALIZATIONS}

Three-dimensional case. Let us consider the three-dimensional domains, where the layers $\Omega_{1}, \Omega_{2}, \ldots$ are cut out from the 3 -D space by the sides of the cone with origin in 0 . The derivatives of discretized functions still contain terms proportional to $1 / r$, but the volumes of elements are proportional to $r^{3}$, so as a result

$E_{i}=\frac{1}{2}\left[\left(u_{i}^{h}\right)^{T},\left(u_{i+1}^{h}\right)^{T}\right] \cdot\left(r^{i-1} M\right) \cdot\left[\begin{array}{l}u_{i}^{h} \\ u_{i+1}^{h}\end{array}\right], \quad i=1,2, \ldots$

with $M$ having the form as in (3). Hence the system $M_{\infty} \cdot Q_{\infty}=B_{\infty}$ becomes

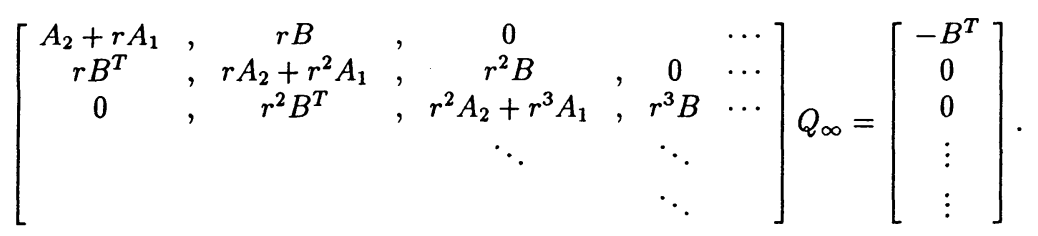

The rows do not repeat here exactly, so the solution requires some scaling. Let $I$ be an $n \times n$ identity matrix, and define $P=\operatorname{diag}\left[r^{-1 / 2} I, r^{-1} I, \ldots, r^{-i / 2} I, \ldots\right]$. Then (16) may be rewritten (diagonal infinite matrix is invertible) as $\left(P^{-1} \cdot \tilde{M}_{\infty} \cdot P^{-1}\right) \cdot Q_{\infty}=B_{\infty}$, where $\tilde{M}_{\infty}=P \cdot M_{\infty} \cdot P$. Moreover, the system $\tilde{M}_{\infty} \cdot \tilde{Q}_{\infty}=\tilde{B}_{\infty}$, where $\tilde{Q}_{\infty}=$ $P^{-1} Q_{\infty}, \tilde{B}_{\infty}=P B_{\infty}$, has the form

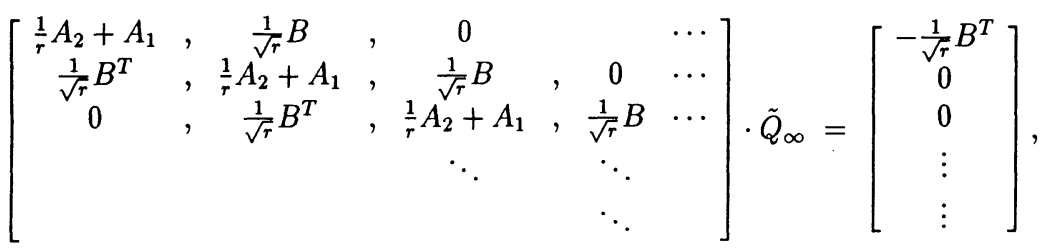


which falls into the framework of our method, with the eigenvalue problem (13)

$\operatorname{det}\left[\lambda^{2} \frac{1}{\sqrt{r}}+\lambda\left(\frac{1}{r} A_{2}+A_{1}\right)+\frac{1}{\sqrt{r}} B^{T}\right]=0$.

The same procedure applies obviously to $1-\mathrm{D}$ and $2 \frac{1}{2}-\mathrm{D}$ domains, only some directions would require different scaling and we would have to define matrix $P$ in a more complicated way, but the idea would remain the same.

Finite domain - superelements. Consider the finite, star-shaped body. We may discretize the boundary and then use radii originating from the star center for the construction of similar layers of domain discretizations with the decreasing size, so now $r<1$. Again, the layers form infinite series filling the body, and all reasoning may be repeated. Thus we get a method for calculating the stiffness matrix for the arbitrary star-shaped super-element. The situation reminds somehow a discrete version of the boundary integral method, since we consider only a thin boundary layer and use the fact, that the unknown function satisfies the state equation inside the domain.

Singular elements. Consider a discretized problem on some domain $\Omega_{h}$ (finite or infinite). Let $p_{0}$ be a point on $\partial \Omega_{h}$ where a geometrical singularity of the solution may occur (reentrant corner, change of the type of boundary conditions), belonging also to the nodes of triangulation. The standard way of dealing with such problems is to refine locally the discretization at the cost of increasing dimensionality (Grisvard,1985). Our method suggests another approach. Create a star-shaped domain consisting of all triangles having $p_{0}$ as a vertex. Then we may treat this star as a finite body and construct for it a stiffness matrix using $p_{0}$ as a similarity origin. In this way we have mesh refinement without dimensionality increase. Moreover, the rate of convergence is the same as in the smooth case.

Improving accuracy. Let us consider the discretization consisting of convex quadrilaterals. Taking centers of these quadrilaterals as similarity centers we may construct stiffness matrices in the same way as for superelements. Such an approach does not increase dimensionality, but, as shown by experiments, improves accuracy in comparison to ordinary linear elements on triangles. This phenomenon occurs in all cases, singular, homogeneous as well as nonsingular and nonhomogeneous.

\section{NUMERICAL EXPERIMENTS}

Infinite domains. In order to test the method, we have conducted several computational experiments involving the known exact solutions to the 2-D Laplace equation. The domain constituted a lower half-plane $y<0$ with the unit circle $x^{2}+y^{2}<1$ cut out. The boundary of this domain can be divided into two parts: $\Gamma_{0}=\{(x, y) \mid(x<$ -1 or $x>1)$ and $(y=0)\}, \Gamma_{1}=\left\{(x, y) \mid(-1 \leq x \leq 1)\right.$ and $\left.\left(y=-\sqrt{\left(1-x^{2}\right)}\right)\right\}$, As test functions we have chosen: [A] $u=x / r^{2}$; [B] $u=\left(y^{2}-x^{2}\right) / r^{4}$. Both of these functions satisfy $\frac{\partial u}{\partial n}=0$ on $\Gamma_{0}$ and their total flux over $\Gamma_{1}$ vanishes, so they also vanish at infinity. The test consisted in imposing a computed (exact) flux along $\Gamma_{1}$ and getting a numerical approximation of $u$. The domain has been discretized into consecutive ring-like layers of growing thickness, the layers themself being cut into $n+1$ 
Table 1 Maximal error along $\Gamma_{1}$ for the case A

\begin{tabular}{lll}
\hline & $\mathrm{n}=16$ & $\mathrm{n}=32$ \\
\hline 2 layers & 0.660 & 0.815 \\
4 layers & 0.396 & 0.643 \\
8 layers & 0.128 & 0.369 \\
16 layers & 0.031 & 0.100 \\
\hline Series app. & 0.025 & 0.006 \\
\hline
\end{tabular}

Table 2 Maximal error along $\Gamma_{1}$ for the case B

\begin{tabular}{lll}
\hline & $\mathrm{n}=16$ & $\mathrm{n}=32$ \\
\hline 2 layers & 0.416 & 0.646 \\
4 layers & 0.158 & 0.374 \\
8 layers & 0.063 & 0.109 \\
16 layers & 0.057 & 0.021 \\
\hline Series app. & 0.057 & 0.016 \\
\hline
\end{tabular}

quadrilaterals by equally spaced radii. The ratio $r$ has been chosen in such a way, that the quadrilaterals resembled squares as well as possible ( so $r$ and the thicknesses of layers depended on $n$ ).

Two methods of the solution have been compared. First, it has been computed by the method presented in this paper and therefore using only the first layer of discretization in order to compute the stiffness matrix $S$. Second, the ordinary FEM solutions have been found over the growing number of layers, with null boundary condition at the outermost boundary of the last layer. The approximation was in both cases linear, i.e. the quadrilaterals have been divided into four triangles on which the linear elements were used, and the internal node was eliminated. The results for the function (A) are shown in Table.1. The fact, that the error of ordinary FEM for $n=32$ approaches its counterpart for series method slower than for $n=16$, is connected with thickness of layers: for $n=32$ they are thinner and therefore 16 layers $(n=32)$ contain smaller area than 16 layers $(n=16)$. The results for function (B) look qualitatively the same. Observe, however, that now the convergence of the ordinary FEM model to the corresponding solutions obtained using our method is faster. The explanation is simple: the function decays faster and more far away layers may be disregarded.

Corner singularity. In this case the test domain constituted a unit circle with one

Table 3 Maximal error and convergence rate for corner singularity

\begin{tabular}{llllc}
\hline & $\mathrm{n}=6$ & $\mathrm{n}=12$ & $\mathrm{n}=24$ & $L_{2}(\Gamma)$-conv. rate \\
\hline FEM & 0.083 & 0.023 & 0.008 & 1.27 \\
Series app. & 0.057 & 0.012 & 0.003 & 1.60 \\
\hline
\end{tabular}


Table 4 Maximal error and convergence rate for homogeneous case

\begin{tabular}{llllc}
\hline & $\mathrm{n}=6$ & $\mathrm{n}=12$ & $\mathrm{n}=24$ & $L_{2}$-conv. rate \\
\hline FEM & 9.79 & 3.34 & 1.03 & 2.25 \\
Series app. & 4.76 & 1.49 & 0.45 & 2.22 \\
\hline
\end{tabular}

Table 5 Maximal error and convergence rate for nonhomogeneous case

\begin{tabular}{llllc}
\hline & $\mathrm{n}=6$ & $\mathrm{n}=12$ & $\mathrm{n}=24$ & $L_{2}$-conv. rate \\
\hline FEM & 0.246 & 0.072 & 0.021 & 2.18 \\
Series app. & 0.197 & 0.049 & 0.013 & 2.17 \\
\hline
\end{tabular}

quarter cut out. On the radii bounding the cut out part the homogeneous Neumann boundary conditions have been imposed. A well known singular solution $u=r^{2 / 3} \cdot \cos \left(\frac{2}{3} \phi\right)$ has been used as a test function (Grisvard,1985). The discretization consisted of $n$ evenly spaced radii and $[n / 3]$ rings, similarly as in former case. We have computed two error indicators: maximal error along the line $\Gamma=r=0.5$ and $L_{2}(\Gamma)$ convergence rate. The star mentioned in the last section consisted of all triangles having a vertex in 0 . The results are summarized in Table 3 . Since convergence rate concerns a projection on a line, theoretically it should be equal to 1.166 ... for ordinary FEM and 1.5 for FEM using local singular elements (Grisvard,1985). As we see, our approach is as good as the second case.

Improving accuracy. Here the computational domain consisted of the square $[0,4] \times$ $[0,4]$ divided into $n \times n$ subsquares. We have compared the performance of the ordinary linear finite element with our modified one for two cases: [A] Test function $u=\exp (x) \sin y$ satisfying homogeneous Dirichlet equation. The results are in Table 4. [B] Test function $u=y^{2} \exp (x)$ satisfying nonhomogeneous Dirichlet equation. The results are in Table 5 . In both cases we have obtained the same rate of convergence as for ordinary FEM, with twice better accuracy.

\section{REFERENCES}

Cooke, R.G. (1950) Infinite Matrices and Sequence Spaces. MacMillan and Co., London. Givoli, D. (1992) Numerical methods for problems in infinite domains. Elsevier, Amsterdam.

Grisvard, P. (1985) Elliptic problems in nonsmooth domains, Pitman, London. 1985.

M. Grote, M. and Keller, J.B. (1994) Nonreflecting boundary conditions. Danish Center for Applied Mathematics and Mechanics Anniversary volume, Technical University of Denmark, (submitted to J. Comput. Phys.).

Sharau, S.K. (1994) Finite element analysis of infinite solids using elastic supports. Computers and Structures, vol. 13, No. 5, 1145-1152.

Stanley, R.P. (1986) Enumerative Combinatorics. Wadsworth \& Brooks/Cole, Monterey, California. 